\title{
Supporting Tourism with Public Interactive Displays
}

\author{
Ville Mäkelä* \\ LMU Munich \\ Munich, Germany \\ ville.maekelae@ifi.lmu.de \\ Mohamed Khamis \\ University of Glasgow \\ Glasgow, United Kingdom \\ mohamed.khamis@glasgow.ac.uk
}

\author{
Vito Gentile \\ Università degli Studi di Palermo \\ Palermo, Italy \\ vito.gentile@unipa.it \\ Salvatore Sorce \\ Università degli Studi di Palermo \\ Palermo, Italy \\ salvatore.sorce@unipa.it
}

\begin{abstract}
Interactive displays are common in public and semi-public areas, such as museums, shopping malls, train stations, and even on streets. Especially with the emergence of new ways of interaction, interactive displays could be introduced to popular tourist attractions to support tourism. The aim of this tutorial is to prepare its participants for designing such interactive public displays. The tutorial will first introduce participants to state of the art in interactive public displays. This will be followed by a city tour where a number of local attractions are visited. Finally, the participants will brainstorm and create concepts for novel interactive public displays that aim to improve the experience of visiting tourist attractions.
\end{abstract}

\section{CCS CONCEPTS}

- Human-centered computing $\rightarrow$ Human computer interaction (HCI); Interaction techniques; Interaction design.

\section{KEYWORDS}

public displays, interaction techniques, tourism, attractions, user experience, landmarks, in-the-wild deployments

\section{ACM Reference Format:}

Ville Mäkelä, Vito Gentile, Mohamed Khamis, and Salvatore Sorce. 2019. Supporting Tourism with Public Interactive Displays. In Proceedings of the 8th ACM International Symposium on Pervasive Displays (PerDis '19), fune 12-14, 2019, Palermo, Italy. ACM, New York, NY, USA, 2 pages. https: //doi.org/10.1145/3321335.3330140

\section{INTRODUCTION}

Interactive displays are a common sight in various public and semipublic locations. These displays are sometimes encountered in tourist attractions such as museums; however, they are much more scarce in certain types of attractions such as architectural marvels. Still, interactive installations have the potential to greatly improve

\footnotetext{
*Also with Tampere University.
}

the experience of visiting tourist attractions, for instance, by offering visitors relevant information and services, or educational or recreational content [5].

Recently, new technologies have brought forth more novel ways of interaction, such as mid-air gestures [9], eye gaze [6], and multimodal approaches $[2,8,11]$. Some creative solutions also combine location tracking with novel interactions $[10,11]$. Moreover, augmented reality can also be combined with interactive displays, for example, through the user's mobile device $[1,3]$.

These developments create opportunities for designing new and creative interactive experiences to support tourism. Such systems can serve as anything from convenient information sources to playful, leisure-oriented activities. Especially from a tourist standpoint, a particularly successful interactive installation may even become an attraction in itself.

The aims of this tutorial are two-layered. First, we aim to give the participants a comprehensive overview of interaction with public displays using various modalities, and provide them with a rich domain in which they can apply the acquired knowledge to design interactive public display applications. Second, we have communal long-term goals: through the observations, brainstorming, and discussions conducted throughout this tutorial, we aim to 1) understand the unique challenges and opportunities that the context of tourism brings to the design and deployment of interactive displays, and 2) produce novel concepts for interactive displays that support tourism. Attending this tutorial does not require prior experience with interactive displays, and because the brainstorming focuses on ideas and concepts, no technical expertise is required.

\section{TUTORIAL STRUCTURE}

The tutorial is split into three parts: an introduction to interactive displays, a city tour to nearby attractions in Palermo, and a handson activity. The detailed program is presented in Table 1.

\subsection{Introduction to Interactive Public Displays}

The tutorial will start with an introduction to public displays, with a focus on novel concepts and the challenges with deploying public displays in the wild. The aim of the introduction is to offer participants sufficient background knowledge to carry out a brainstorming task later on. The primary covered topics are as follows:

Design space of interactive public displays. This topic will cover the seminal research on public displays and audience behavior, such as how people behave around public displays [4], what 
Table 1: Program.

\begin{tabular}{l|l} 
Time & Activity \\
\hline 09:00 - 09:15 & Introducing speakers and participants \\
09:15 - 09:45 & Introduction to public displays research \\
09:45 - 10:15 & Interactive displays \\
10:15 - 10:45 & Evaluating public displays \\
10:45-11:00 & Coffee break \\
11:00-12:30 & Tour around Palermo \\
\hline 12:30-13:30 & (Working) Lunch \\
\hline 13:30- 15:00 & Hands on activity \\
15:00 - 16:00 & Participants' presentations of outcomes \\
16:00- 16:15 & Closing activities
\end{tabular}

factors are involved, and how displays must attract and motivate passersby [13]. We also touch on what challenges lie in designing and deploying interactive installations in the wild, for instance, how they are affected by external factors [12].

Interacting with public displays. We will present state of the art research in designing interactions for pervasive public displays. We will focus on novel interactions, such as mid-air gestures, gaze, and multimodal interaction where two or more modalities are combined. We will discuss opportunities and challenges that these modalities bring [7].

\subsection{Visiting Palermo's Tourist Attractions}

The participants will be then guided in a city tour where they will visit various popular attractions in Palermo. The aim of the tour is to serve as both a knowledge-gaining experience as well as a source of inspiration. Participants are encouraged to observe if and what kind of interactive displays exist to serve the visitors to the attractions, and how they could be improved. In particular, participants should think about how both the attractions and the visitors could benefit from novel interactive concepts, and also, what kinds of unique challenges the attractions present with regards to deploying interactive technology around them.

\subsection{Concept Production and Feedback}

Building on the knowledge gained in the introduction and drawing upon the inspiration from the city tour, the participants will then sketch concepts for prototype applications that would support tourism at said attractions. The focus is on the generation of ideas and concepts - not on technical contributions.

At the end of the tutorial, the concepts will be presented to the other attendees for feedback. Participants will be encouraged to later implement their concepts and follow up the work with an evaluation to create a scientific contribution (e.g., that can be a start for a paper at PerDis 2020).

\section{OBJECTIVES AND EXPECTED OUTCOMES}

During this tutorial, our communal objectives are as follows:

- Objective 1: Dissemination of knowledge: through this tutorial, participants should have a general understanding of the possibilities and challenges in the context of interactive displays.
- Objective 2: Identification of the unique challenges and opportunities in the design and deployment of interactive displays that support tourism.

- Objective 3: Production of novel concepts for interactive systems that aim to support tourism.

The concepts produced through this tutorial have the potential to lead to fully realized research projects in the future.

\section{ACKNOWLEDGMENTS}

This work was partly supported by the Ulla Tuominen Foundation and the Foundations' Post Doc Pool.

\section{REFERENCES}

[1] Salvatore Andolina, Dario Pirrone, Giuseppe Russo, Salvatore Sorce, and Antonio Gentile. 2012. Exploitation of Mobile Access to Context-Based Information in Cultural Heritage Fruition. In 2012 Seventh International Conference on Broadband, Wireless Computing, Communication and Applications. IEEE. https://doi.org/10. 1109/bwcca.2012.60

[2] Agnese Augello, Antonella Santangelo, Salvatore Sorce, Giovanni Pilato, Antonio Gentile, Alessandro Genco, and Salvatore Gaglio. 2007. A Multimodal Interaction Guide for Pervasive Services Access. In IEEE International Conference on Pervasive Services. IEEE. https://doi.org/10.1109/perser.2007.4283923

[3] Matthias Baldauf and Peter Fröhlich. 2013. The Augmented Video Wall: Multiuser AR Interaction with Public Displays. In CHI '13 Extended Abstracts on Human Factors in Computing Systems (CHI EA '13). ACM, New York, NY, USA, 3015-3018. https://doi.org/10.1145/2468356.2479599

[4] Vito Gentile, Mohamed Khamis, Salvatore Sorce, and Florian Alt. 2017. They Are Looking at Me!: Understanding How Audience Presence Impacts on Public Display Users. In Proceedings of the 6th ACM International Symposium on Pervasive Displays (PerDis '17). ACM, New York, NY, USA, Article 11, 7 pages. https: //doi.org/10.1145/3078810.3078822

[5] Vito Gentile, Salvatore Sorce, Giuseppe Russo, Dario Pirrone, and Antonio Gentile. 2016. A Multimodal Fruition Model for Graphical Contents in Ancient Books. In Proceedings of the 17th International Conference on Computer Systems and Technologies 2016 - CompSysTech '16. ACM Press. https://doi.org/10.1145/2983468. 2983477

[6] Mohamed Khamis. 2018. Designing gaze-based interaction for pervasive public displays. Ph.D. Dissertation. Ludwig Maximilian University of Munich (LMU), Geschwister-Scholl-Platz 1, 80539 Munich, Germany. http://nbn-resolving.de/ urn:nbn:de:bvb:19-227815

[7] Mohamed Khamis, Florian Alt, and Andreas Bulling. 2016. Challenges and Design Space of Gaze-enabled Public Displays. In Proceedings of the 2016 ACM International foint Conference on Pervasive and Ubiquitous Computing: Adjunct (UbiComp '16). ACM, New York, NY, USA, 1736-1745. https://doi.org/10.1145/ 2968219.2968342

[8] Mohamed Khamis, Daniel Buschek, Tobias Thieron, Florian Alt, and Andreas Bulling. 2018. EyePACT: Eye-Based Parallax Correction on Touch-Enabled Interactive Displays. Proc. ACM Interact. Mob. Wearable Ubiquitous Technol. 1, 4, Article 146 (Jan. 2018), 18 pages. https://doi.org/10.1145/3161168

[9] Ville Mäkelä, Tomi Heimonen, Matti Luhtala, and Markku Turunen. 2014. Information Wall: Evaluation of a Gesture-controlled Public Display. In Proceedings of the 13th International Conference on Mobile and Ubiquitous Multimedia (MUM '14). ACM, New York, NY, USA, 228-231. https://doi.org/10.1145/2677972.2677998

[10] Ville Mäkelä, Jobin James, Tuuli Keskinen, Jaakko Hakulinen, and Markku Turunen. 2017. "It's Natural to Grab and Pull": Retrieving Content from Large Displays Using Mid-Air Gestures. IEEE Pervasive Computing 16, 3 (2017), 70-77. https://doi.org/10.1109/MPRV.2017.2940966

[11] Ville Mäkelä, Mohamed Khamis, Lukas Mecke, Jobin James, Markku Turunen, and Florian Alt. 2018. Pocket Transfers: Interaction Techniques for Transferring Content from Situated Displays to Mobile Devices.. In Proceedings of the 36th Annual ACM Conference on Human Factors in Computing Systems (CHI '18). ACM, New York, NY, USA, 13. https://doi.org/10.1145/3173574.3173709

[12] Ville Mäkelä, Sumita Sharma, Jaakko Hakulinen, Tomi Heimonen, and Markku Turunen. 2017. Challenges in Public Display Deployments: A Taxonomy of External Factors. In Proceedings of the 2017 CHI Conference on Human Factors in Computing Systems (CHI '17). ACM, New York, NY, USA, 3426-3475. https: //doi.org/10.1145/3025453.3025798

[13] Jörg Müller, Florian Alt, Daniel Michelis, and Albrecht Schmidt. 2010. Requirements and Design Space for Interactive Public Displays. In Proceedings of the 18th ACM International Conference on Multimedia (MM '10). ACM, New York, NY, USA, 1285-1294. https://doi.org/10.1145/1873951.1874203 